\title{
A Combined Neuro Fuzzy-Cellular Automata Based Material Model for Finite Element Simulation of Plane Strain Compression
}

\author{
S. Das $*^{2}$, M.F. Abbod*, Q. Zhu*¹, E.J. Palmiere`, I.C. Howard* and D.A. Linkens ${ }^{\star}$
}

\section{IMMPETUS}

Institute for Microstructural and Mechanical Process Engineering, The University of Sheffield

\author{
^School of Engineering and Design, Brunel University, Uxbridge UB8 3PH \\ •Department of Automatic Control and Systems Engineering, \\ -Department of Engineering Materials \\ *Department of Mechanical Engineering \\ University of Sheffield, Sheffield S1 3JD, UK
}

Corresponding Author:

Dr M.F. Abbod

Howell Building,

School of Engineering and Design,

Brunel University, Uxbridge UB8 3PH, United Kingdom

Tel: +44-(0)1895267061

email: maysam.abbod@brunel.ac.uk

\footnotetext{
${ }^{1}$ Current address: Holset Engineering Co. Ltd., St Andrew's Road, Huddersfield HD1 6RA, UK.
}

${ }^{2}$ Current address: Technologist, Automation Division, Tata Steel, Jamshedpur - 831001 India. 


\begin{abstract}
:
This paper presents a modelling strategy that combines Neuro-Fuzzy methods to define the material model with Cellular Automata representations of the microstructure, all embedded within a Finite Element solver that can deal with the large deformations of metal processing technology. We use the acronym nf-CAFE as a label for the method. The need for such an approach arises from the twin demands of computational speed for quick solutions for efficient material characterisation by incorporating metallurgical knowledge for material design models and subsequent process control. In this strategy, the cellular automata hold the microstructural features in terms of sub-grain size and dislocation density which are modelled by a neuro-fuzzy system that predicts the flow stress. The proposed methodology is validated on a two dimensional (2D) plane strain compression finite element simulation with Al-1\% Mg alloy. Results from the simulations show the potential of the model for incorporating the effects of the underlying microstructure on the evolving flow stress fields. In doing this, the paper highlights the importance of understanding the local transition rules that affect the global behaviour during deformation.
\end{abstract}

Keywords: Cellular Automata, Neuro-Fuzzy, Finite Element, Microstructure Modelling, 


\section{Introduction}

Thermo-mechanical simulations of metal-forming operations typically use the finite element (FE) method to study the evolution of deformation variables e.g. stress, strain and temperature. The relationship between the externally applied deformation and the internally evolving variables can be captured by a material model that is expressed by physically based equations. Such physically-based relations [1][2] use the fundamental variables of dislocation density $(\rho)$, sub-grain size $(\delta)$ and misorientation angle $(\theta)$ to capture the local hardening and softening phenomena based on the externally applied deformation. The local effects are summed at the global structural level to arrive at the structural stress patterns.

Recently, physically-based formulations have utilised the merits of Artificial Intelligence (AI) tools, particularly the concept of Neuro-Fuzzy (NF) architectures, to define the material model [3][4]. NF models work well in situations where the experimental data on the evolution rates of $\rho, \delta$ and $\theta$ are sparse or difficult to obtain for the entire workable range of deformation conditions. To carry out simulation studies at the structural level, the physically-based formulations need to be coded insitu within the finite element solver using user-subroutines.

A particular area of interest is the effect of the initial microstructure on the evolution rates of $\rho, \delta$ and $\theta$. Experimental evidence shows that the initial distribution of grain size, particularly the grain boundaries, play an important role in defining the evolution rules. An efficient method of capturing the initial and evolving microstructural information is to use a combination of cellular automata (CA) and finite element (FE) techniques, a result often termed CAFE [5][6][7]. It uses the scale-invariant spatial 
characteristic of CA to define the micro-feature and it links the CA cells to the integration point of a finite element.

The concept of Cellular Automata was introduced by von Neumann [13]in the early 1940's for the simulation of self-reproducing automata and population evolution. Early developments in application were to the fields of fluid dynamics and biological processes, and recently it has been applied to the simulation of material microstructure [14][15][16].

In the CAFE framework, the material model is defined by the rate of evolution of $\rho, \delta$ and $\theta$ at each time increment. The rate constants used in the evolution equations of $\rho$, $\delta$ and $\theta$ are based on a neuro-fuzzy model which has been developed using the experimental work reported by Sellars and Zang [10].

This paper reports the concept and development of this new combined neuro-fuzzy and cellular automata based material model. A schematic illustration of this approach is shown in Figure 2 for a single element highlighted in this concept. The material model has been encoded in the user subroutine USDFLD within the finite element software ABAQUS ${ }^{\mathrm{TM}}$ which was used to study the evolution of the stress patterns at specific spatial locations in a model of $2 \mathrm{D}$ plane strain compression of a $\mathrm{Al}-1 \% \mathrm{Mg}$ alloy. At each time increment of the solver, the FE integration point provides the strain, strain-rate and temperature to the user-subroutine. These variables are distributed using simple rules to accommodate the variances of the underlying microfeatures. For the case illustrated in Figure 2, the strains at the grain boundaries and interiors are designated as $\varepsilon_{g b}$ and $\varepsilon_{i n}$. The strains, their rates and temperature 
variables are passed to the NF material model to calculate the current value of the flow stress for each individual CA cell, then the summation of all the stresses from each CA cell is obtained to present the final stress of the FE cell. The paper shows that subtle changes in the initial distribution of microstructural features affect the evolution of the stress patterns and this also varies from point to point within the deforming model.

\section{Experimental Observations}

During thermomechanical processing of materials such as aluminium alloys, local regions deform under conditions that involve continuous change in strain-rate and temperature. Decreasing strain-rate and changing straining direction have a significant effect on the evolution of deformation microstructure, texture and any subsequent recrystallisation behaviour [1][8]. Microbands, as a dislocation substructure, form during deformation both at room and high temperature in aluminium. The structure is created by geometrically-necessary dislocations that form walls leading to in-grain lattice curvatures. The microbands are initially evolved at low strains when a single or a few slip systems are active, and then become cell blocks or equiaxed subgrains. The geometrically-necessary boundaries have higher misorientation angles across them than other dislocation sub-boundaries, and have a significant effect on subsequent recrystallisation behaviour. During rolling or plane strain compression (PSC) deformation, the microbands form parallel plates oriented at an average $35^{\circ}$ to the surface or the effective rolling direction. 
Zhu et al [1] and Sellars and Zhu [9] state that the mechanism of the effect arises from the different evolution of the geometrically-necessary dislocation during monotonic and reversed deformation, or during changes in strain-rate. The dislocation density within sub-boundaries $\rho_{\mathrm{b}}$ increases with strain during monotonic deformation as the spacing between the sub-boundaries $\delta$ decreases and misorientation $\theta$ across the subboundaries increases according to the relationship $\rho_{\mathrm{b}}=C \theta / b \delta$, where $C(\approx 1-2$ depending on the type of the sub-boundaries) is a geometrical parameter. On the other hand, the dislocation density decreases with strain after a change in the straining direction or a decrease in the strain-rate.

The dislocation density initially increases with plastic deformation until the stage where the increase in dislocation density arising from strain hardening and the decrease arising from dynamic recovery become dynamically balanced. Then the dislocation density remains constant with further deformation. When dynamic recovery becomes significant, substructures such as dislocation cells/sub-grains or micro-bands form. Experimental observations show that the sub-grain size decreases but the misorientation between sub-grains increases with plastic deformation. Figure 3 shows three typical dislocation substructures (Types 1,2 and 3) that occur during thermomechanical processing. Hansen and Huang [10] determined that the three different dislocation substructures have coherent relationships with the orientation of the grains, as shown in Figure 4. The dislocation substructures represented by Type 1 have grains with orientations to the stress axis concentrated in the region of the lower half, close to [100], with Taylor factor less than 3.0; Type 2 have grains with orientations concentrated in the region close to [100] with the Taylor factor less than 
2.5; and Type 3 have grains distributed over the remaining area of the triangle (Figure $4 a)$.

\section{Material Characteristics Model}

The neuro-fuzzy model was developed to predict the parameters of the material microstructure $(\rho, \delta$ and $\theta)$ that are used to calculate the stress. The relations are as follows.

$$
\begin{gathered}
\sigma_{\rho i}=\alpha_{1} M G b \rho_{i}^{1 / 2} \\
\sigma_{\delta}=\alpha_{2} M G b / \delta \\
\sigma_{f}=\frac{M B}{D_{0} b^{2}} \cdot \frac{Z}{\rho_{m}} \\
\sigma_{i}=\sigma_{\rho i}+\sigma_{\delta}+\sigma_{f p}
\end{gathered}
$$

where the constants $\alpha_{1}=0.38$ and $\alpha_{2}=0.79$ for aluminium alloys, the Taylor factor for polycrystals is $M=3, D_{0}=1$ is the diffusion frequency, $G=(29484-13.6 \mathrm{~T}) \mathrm{MPa}$ where $\mathrm{T}$ is the temperature is the shear modulus, $b=0.286 \times 10^{-9} \mathrm{~m}$ is the Burgers vector, $\rho_{\mathrm{m}}$ is the mobile dislocation density, (which is the same order of magnitude as the internal dislocation density $\rho_{\mathrm{i}}$ for hot deformation at constant strain rate) and $\mathrm{B}$ is a material constant reflecting solution hardening.

In the NF model, the inputs are the deformation conditions of strain rate, temperature and deformation level (i.e. strain for a given material) [11][12]. 
Sample results for modelling the internal dislocation density, sub-grain size and misorientation for a constant strain rate $(2.5 / \mathrm{s})$ and changing strain rates are shown in Figure 5. The solid line is the modelled output, while the dotted points are the experimental data.

There is no explicit inclusion of the initial spatial grain structure in the approach outlined above. The representation is in terms of a mean grain size that is used to evaluate the internal state variables in the NF program. This does not deal particularly well with important features in material processing, where the evolution of microstructural features can be very inhomogeneous. What is more, such microstructural variance can affect the local resistance to further deformation. We need, therefore, a way of coupling these effects.

\section{The Cellular Automata / Finite Element (CAFE) Framework}

The CAFE framework uses cellular automata cells to capture the important microstructural details. It embeds this information within the integration point of an

overlying finite element. The microstructural details are those characterised by the material region surrounding the CA cell and are defined in terms of logical states e.g. grain interiors and grain boundaries or lumped quantities of average dislocation densities. The integration is brought about with the help of user subroutines within ABAQUS. A more detailed account of the technique can be found in reference [17].

The CAFE framework for a plane strain compression model is formulated below. The workpiece geometry in plane strain compression is discretised using finite elements of 
the order of millimetres $(\sim 0.5-1 \mathrm{~mm})$. Figure 6 a illustrates one such discretisation where the domain is divided into 10 finite elements.

Each finite element is populated with CA cells, the size of which are suitably adjusted to the feature of interest. The CA cell associates with itself a material region around itself. All properties (intrinsic or extrinsic) are homogenous over this material region.

The simulation of a representative microstructure starts by populating these cells with pre-specified numbers of nuclei. Each nucleus is assigned a tag that defines its spatial position and its orientation with respect to a fixed global Cartesian coordinate system (Figure 6b). The orientation is illustrated in Figure 6c for nuclei N1 and N2 with orientation angles $\mathrm{t} 1$ and $\mathrm{t} 2$ with respect to the $\mathrm{X}$ Cartesian axis. All other cells have null orientation. The orientation variable varies between zero and $360^{\circ}$.

A suitable neighbourhood is then selected that defines the probable direction of the moving fronts emerging from the nuclei. The neighbourhood of a cell is usually taken to be the cell itself and the cells sharing an edge or a corner of the centre cell. If the cell currently being computed is represented as A (Figure 6d), then using Moore's definition of a CA neighbourhood, the cells represented by tpA, btA, leA, rtA influence the moving front. The state of cell A is modified based on the states of the surrounding cells. The velocity of the moving front of each nucleus is converted into the spatial dimension ( $d X$ or $d Y$ ) moved per unit time. At each step, the nuclei will spread over cells that have a null orientation. This is true for both directions. Growth is interrupted when the moving fronts of two nuclei meet. This interface forms the grain boundary having a misorientation defined by the difference of orientations 
across the boundaries. This is the form of Figure $6 \mathrm{e}$ and is the microstructure linked to the integration point of an overlying finite element.

Once the grain interiors and grain boundary representation is complete, each CA cell is associated with an initial dislocation density that varies between pre-specified upper and lower bounds. Theoretically, the lower bound is equal to zero i.e. the material region is free from dislocations. Experimentally, however, dislocation densities are of the order of $10^{12} / \mathrm{m}^{2}\left(=10^{6} / \mathrm{mm}^{2}\right)$ even in well-annealed materials.

Figure $7 \mathrm{a}$ shows the simplest construction of a microstructure consisting of nine grains having four different orientations corresponding to (110), (111), (100) and (112). At this stage of 2D model formulation, these are numbers that reduce to angles of $0,30,45$ and 90 degrees with the principal straining direction. The constructed microstructure is designed to have similar orientations to the experimentally observed $\mathrm{Al}-1 \% \mathrm{Mg}$ microstructure (Figure $7 \mathrm{~b}$ ) where the different grey levels highlight different orientations.

This concept was extended to form coarse (Figure 8a) and fine (Figure 8b) microstructures containing approximately 100 and 300 grains respectively. For these grains, the orientation distributions are shown in Figure 9a. For the simulations that follow, these orientation distributions can be classified into biased and non-biased regions (shaded portions in Figure 9b). The intention was to demonstrate the potential of the modelling strategy to account for differences in internal response to the applied strain direction. The biased regions offer a greater resistance to applied strain and therefore harden faster than the non-biased regions. 
A similar approach was used for the CA cells that form part of the grain boundary and those that form part of the grain interior. Those CA cells forming part of the grain boundary were allocated a higher strain (as is consistent with the assumption that grain boundaries act as dislocation sinks) and those within the grain boundary were ascribed the same values as the macroscopic strain at the integration point of the element. The NF program was then called to evaluate the stress in terms of the internal variables of strain, strain-rate and temperature at each CA cell.

\section{Model Assembly}

Figure 10 shows the PSC finite element model where both the specimen and tool are modelled using 4-noded quadrilateral elements. All the stock elements were initialised with a starting temperature of $400^{\circ} \mathrm{C}$ and the tool elements at $390^{\circ} \mathrm{C}$. Friction at the tool-stock interface was modelled using the Amonton-Coulomb law with a constant coefficient of friction of 0.1 . The heat transfer coefficient at the interface was 80 $\mathrm{kW} / \mathrm{m}^{2} \mathrm{~K}$. The instantaneous tool velocity changes with the instantaneous specimen height and was controlled to achieve a constant nominal strain-rate of $3 / \mathrm{s}$. The stock thickness was reduced by $50 \%$ during the deformation.

The simulation began with the introduction of a representative microstructure into each finite element. The first simulation set is one in which each finite element constituting the element set $\mathrm{A}$ is populated with the simplified microstructure of Figure $7 \mathrm{a}$ in which only 4 orientations are permitted. 
The second simulation set is one in which each element constituting the element set A is populated with the representative microstructure of Figure 8.

\section{Results and Discussions}

\subsection{Qualitative Shape Changes}

Figure 11a shows the deformation pattern of the nine grains constituting the simplified microstructure of Figure 7. The grains are elongated along the compression direction. The reasonableness of the combined modelling approach is evident from a qualitative comparison between the simulated deformed microstructure of Figure 11a and the experimentally observed microstructure of $\mathrm{Al}-1 \% \mathrm{Mg}$ alloy of Figure $11 \mathrm{~b}$.

Figure 12 shows the grain structure in element number 3 located at the bottom-left hand corner of the specimen (third from bottom of element set A (shaded)). The element was $100 \mathrm{~mm}$ by $100 \mathrm{~mm}$ in size before deformation, and it changed to 200 $\mathrm{mm}$ by $56 \mathrm{~mm}$. The grain structure at the end of the test as simulated using the $n f$ CAFE model is shown by the grey dots. Comparing the grain structures before and after deformation shows a qualitatively reasonable change in grain shape. This change in grain shape is associated with evolution of dislocation structures and increase in volume fraction of grain boundary surface.

\subsection{Effect of biasing grain boundaries}

In the above simulation, the $n f$-CAFE formulation was called at each CA cell and assumed the same integration point strain at both grain interiors and grain boundaries.

Changing this distribution affects the predictions. Figure 13 displays the effect of biasing the strain distribution towards the grain boundaries, using bias features of 1.5 and 3.0. With a 1.5 bias, the difference is less than $1 \mathrm{MPa}$. This rises to around $3 \sim 4$ 
MPa with a bias of 3.0. This is because the underlying microstructural feature at each CA cell forms the basis to initialise and evolve its local dislocation density. In other words, if a CA cell belongs to a grain boundary, a biasing factor increases its dislocation storage in terms of CA strain. Results show that a variance of around $50 \%$ does not affect the global stress patterns. It is only after a biasing of more than $150 \%$ that a noticeable change is observed.

\subsection{Effect of grain sizes}

Figure 14 displays the stress predictions for a change in the initial grain sizes, one with large grains and the other with small grains. There is an insignificant difference of about $0.5 \mathrm{MPa}$. The distributions are for the two initial microstructures of Figure 8a and $8 b$.

\subsection{Effect of Orientation Biasing}

The effect of orientation biasing on the evolving von Mises stress fields is shown in Figure 15. In Figure 15a, no biasing is allowed. In Figure 15b, all regions falling outside the shaded area of Figure $9 \mathrm{~b}$ are biased with a factor of $20 \%$. This means that these regions with these orientations should harden faster and this is evident in the higher stress levels of Figure 15b.

Additionally, there are two regions that are of interest. The letters A and B denote regions compressed by the centre of the tool and by the edge, respectively. A superscript star $(*)$ denotes the model with biasing, whilst an absence indicates none. In region $\mathrm{A}$, near the centreline, the stress changes by about $4 \mathrm{MPa}$ with biasing. It has a value of $54 \mathrm{MPa}$ in Region $\mathrm{A}^{*}$, but only in $50 \mathrm{MPa}$ in Region $\mathrm{A}$. In the edgeregion, $\mathrm{B}$, biasing affects not only the value of the stress, but also its distribution, as should be clear from Figure 15. 


\section{Conclusions}

The paper has presented a generic method for representing the microstructure of deformed metals within the continuum formulation of finite element structural modelling. The $n f$-CAFE framework was applied to model the behaviour of the materials during hot deformation using a hybrid modelling technique. Sensitivity studies of the initial grain size of the material were conducted. The $n f$-CAFE model is based on the internal states and predicts the deformed material properties. The results of the $n f$-CAFE numerical models generally compare well with those of models generated using empirical equations, and their predictions are more accurate in characterising deformation at the initial and final stages of the deformation.

The material behaviour using the $n f$-CAFE approach was based on the physicallybased relations that relate the stress evolution to the total dislocation density and the sub-grain size. A major benefit of this modelling technique is the modelling of the dynamic evolution of the internal states, while still providing accurate final material properties in terms of stress/strain relationships. A second advantage is the ability of the model to predict the material properties at a micro-scale without the need for the high computational burden that would be imposed by reducing the mesh size of a straightforward macro-micro finite element model.

The CA technique can analyse the FE cell further into finer cellular structures without changing the meshing scale. Furthermore, one of the advantages of the neuro-fuzzy model as used here is the ability to predict the recrystallisation behaviour based on the 
material microstructure ( $\rho$ and $\delta$ ) at a CA level. This latter feature is currently under development.

\section{Acknowledgment}

The authors gratefully acknowledge the Engineering and Physical Sciences Research Council, UK for their financial support under grant no. GR/L50198.

\section{References}

[1] Q. Zhu, C.M. Sellars, Effect of Deformation Paths on Static Recrystallisation Behaviour of an Al-2Mg, Recrystallisation and Related Topics REX'96, eds. T.R. McNelley et.al, Monterey, California, USA, October (1996) 195-202.

[2] K. Marthinsen, E. Nes, A General Model For Metal Plasticity, Materials Science and Engineering, A234-236 (1997) 1095-1098.

[3] M.F. Abbod, D.A. Linkens, Q. Zhu, Semi-Physical Modelling of Internal States for Aluminium Alloys, Materials Science \& Engineering A, A333 (2002), 397408.

[4] M.F. Abbod, J. Talamantes-Silva, Q. Zhu, D.A. Linkens, Modelling of Aluminium Rolling Using Finite Elements and Grey-Box Modelling Technique, Proceedings of the 2002 IEEE International Symposium on Intelligent Control (ISIC2002), Vancouver, British Columbia, Canada, 27-30 October, eds. C.W. de Silva and F. Karray, The publisher: IEEE Control Systems Society (2002) $321-326$.

[5] S. Das, E.J. Palmiere, I.C. Howard, CAFE: A New Approach to the Modelling of Multipass Hot Rolling, Symposium on Metal Rolling Processes, IOM, London, (2001). 
[6] S. Das, E.J. Palmiere, I.C. Howard, CAFE: A Tool For Modelling Thermomechanical Processes, Proceedings of an International Conference on Thermomechanical Processing: Mechanics, Microstructure, Control. ed. Palmiere, Mahfouf and Pinna. The University of Sheffield, UK (2003) 296-301.

[7] S. Das, E.J. Palmiere, I.C. Howard, Modelling Recrystallisation During Thermomechanical Processing Using CAFE, Materials Science Forum, 467-470 (2004) 623-628.

[8] Q. Zhu, M.F. Abbod, C.M. Sellars, D.A. Linkens, Physically-based and NeuroFuzzy Hybrid Modelling of Aluminium Alloys During Thermomechanical Processing , Conference of Metallurgists CIM (COM 2002), August 11-14, 2002, Montreal, Canada.

[9] C.M. Sellars, Q. Zhu, Microstructural Modelling of Aluminium Alloys during Thermomechanical Processing, Materials Science and Engineering, A280 (2000) 1-7.

[10] N. Hansen, X. Huang, Microstructure and Flow Stress of Polycrystals and Single Crystals, Acta Mat., 46, 5 (1998) 1827-1836.

[11] Q. Zhu, M.F. Abbod, J. Talamantes-Silva, C.M. Sellars, D.A. Linkens, J.H. Beynon, Hybrid Modelling of Aluminium-Magnesium Alloys During Thermomechanical Processing in terms of Physically-Based, Neuro-Fuzzy and Finite Element Models, Acta Mat , 51 (2003) 5051-5062.

[12] C.M. Sellars, M.F. Abbod, Q. Zhu, D.A. Linkens, Hybrid Modelling Methodology Applied To Microstructural Evolution During Hot Deformation Of Aluminium Alloys,, Int. Conf. on Processing and Manufacturing of Advanced Materials (THERMEC’2003), Madrid, Spain, 7-11 July, (2003) 2734. 
[13] J. von Neumann, The general and logical theory of automata, in W. Aspray and A. Burks, editors, papers of the John von Neumann on Computing and Computers Theory, vol 12 in the Charles Babbage Institution Reprint Series for the History of Computing, 1987, MIT Press (1963).

[14] D. Raabe, Computational Materials Science, Wiely-VCH, Weinheim (1998).

[15] D. Raabe, Cellular Automata for materials science with particular reference to recrystallisation simulation, Annual Review of Materials Research, 32 (2002), $53-76$.

[16] D. Raabe, R.C. Becker, Coupling of plasticity finite-elements model with a probabilistic cellular automation for simulating primary static recrystallisation in aluminium. Modelling and Simulation in Materials Science Engineering, 8 (2000), 445-462.

[17] S. Das, A. Shterenlikht, I. C. Howard and E. J. Palmiere, A general method for coupling microstructural response with structural performance, Proceedings of the Royal Society of London, A, (2006), 462, 2085 - 2096. 


\section{List of Figures}

Figure 1: Schematic diagram of the $n f$-CAFE model.

Figure 2: The physically-based model formulation and its linkage to each integration point of a finite element. However, the linkage is made at each CA cell and its state, i.e. whether it is a grain boundary or a grain interior.

Figure 3: Dislocation substructures in Al-\% Mg during thermomechanical processing: (a) elongated microbands after deformation at $300^{\circ} \mathrm{C}$ and $0.1 / \mathrm{s}$ to a strain of 0.3 in axisymmetric tension, (b) cells within elongated microbands after deformation at $385^{\circ} \mathrm{C}$ and $2.5 / \mathrm{s}$ to a strain of 0.7 in plane strain compression and (c) equiaxed subgrains after deformation in plane strain compression at $385^{\circ} \mathrm{C}$ and $2.5 / \mathrm{s}$ to a strain of unity in plane strain compression testing. (b \& c) samples are taken from the maximum deformation zone, while in (a) a homogenous tension deformation means the sample can be taken from anywhere.

Figure 4: Dislocation substructures of pure aluminium after room temperature tension at a strain rate of $8.3 \times 10^{-4} / \mathrm{s}$ : (a) Inverse pole figure showing the tensile axis orientation of 89 grains embedded in polycrystalline specimens strained in the range 0.05-0.34. (b) Type 1 represents the grains with crystallographic dislocation boundaries, (c) type 2 the grains containing equiaxed dislocation cell/sub-grains and (d) type 3 the grains with non-crystallographic dislocation boundaries.

Figure 5: Internal states evolution using the neuro-fuzzy model prediction for Al$1 \% \mathrm{Mg}$ material.

Figure 6: The three domains of the present CAFE model. (a) The structure domain divided into a number of finite elements; one element is shown with its expected array of cells. (b) A population of nuclei in a CA array from which the grain will grow. (c) Definition of orientations with respect to the loading direction. (d) Moore's definition of a CA neighbourhood. (e) A grain structure that emerges from the nuclei of (b).

Figure 7: Simple microstructure with 4 orientations.

Figure 8: Representative microstructures with (a) 100 grains forming a coarse microstructure. (b) 300 grains forming a fine microstructure.

Figure 9: (a) Orientation distributions for each nucleus used to generate the microstructure of Figure 7. (b) Highlighted region shows biasing of specific orientations with respect to a particular loading direction.

Figure 10: Finite element model assembly for the PSC tests.

Figure 11: The simple microstructure of figure 3 after deformation.

Figure 12: Change in microstructure shape for element no. 3, before and after deformation. 
Figure 13: von Mises stress distribution to show the effect of strain biasing at grain boundaries using the nf-CAFE methodology

Figure 14: Comparison of nf-CAFE model prediction with a change in the grain size.

Figure 15: Comparison of nf-CAFE model prediction with introduction of orientation biasing. (a) No Biasing. (b) With a biasing of $20 \%$. 


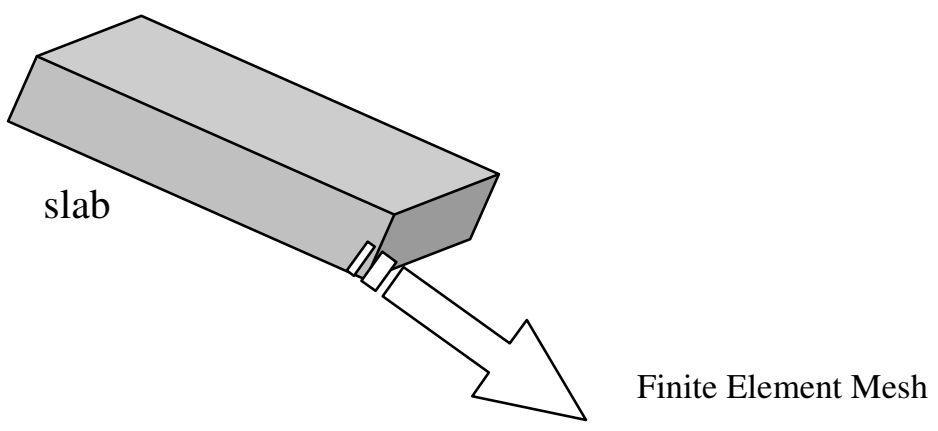

Neuro-Fuzzy Model

inputs: deformation conditions

(T, $\varepsilon, \mathrm{d} \varepsilon / \mathrm{dt})$

output: microstructure $(\rho, \delta$,

$\theta$ )and properties $(\sigma$, drex)

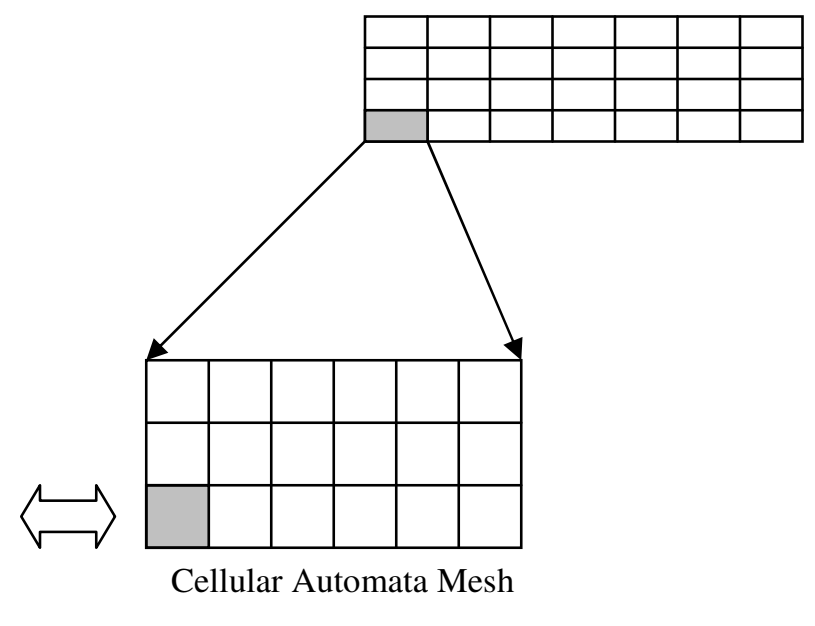

Figure 1: Schematic diagram of the nf-CAFE model. 


\section{FE}

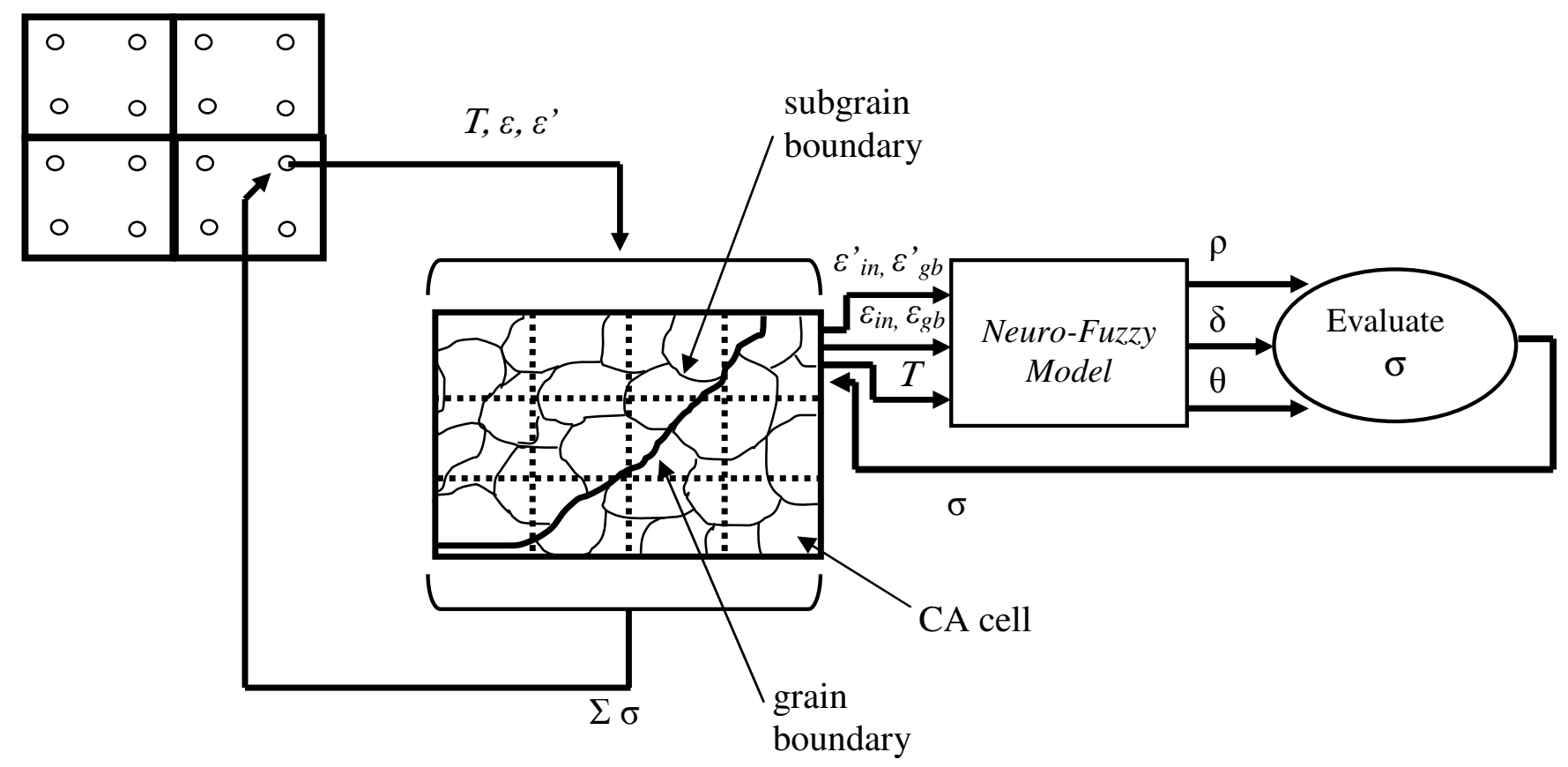

Figure 2: The physically-based model formulation and its linkage to each integration point of a finite element. However, the linkage is made at each CA cell and its state, i.e. whether it is a grain boundary or a grain interior. 
(a)

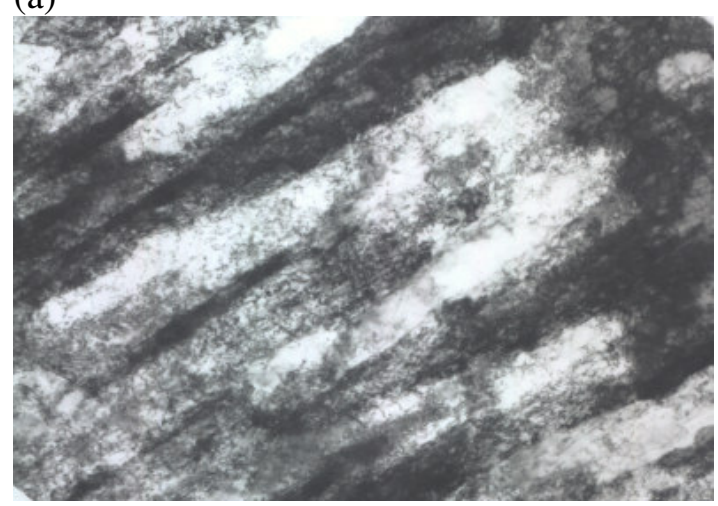

(c)

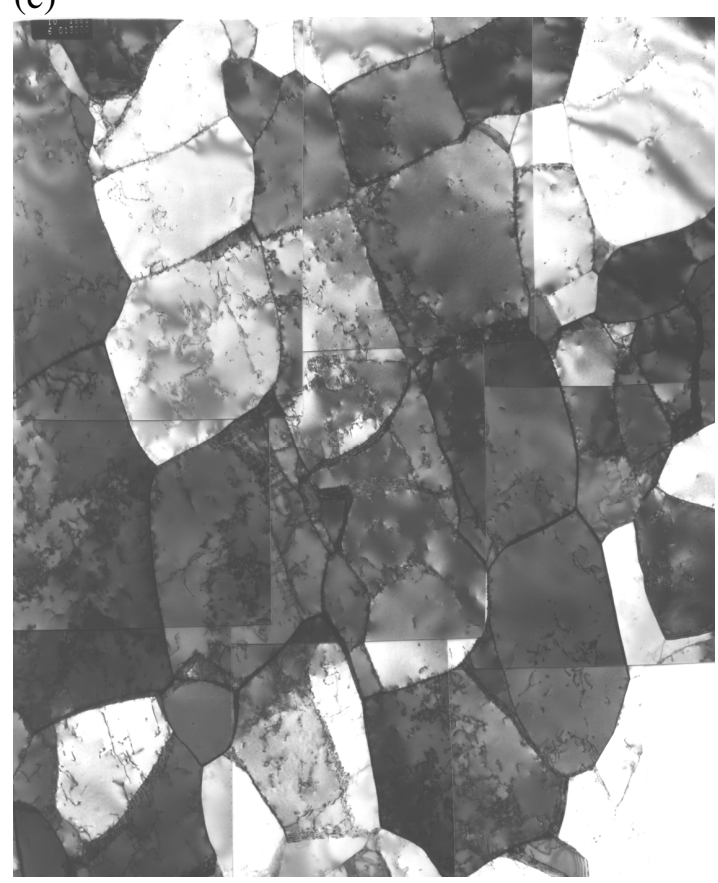

(b)

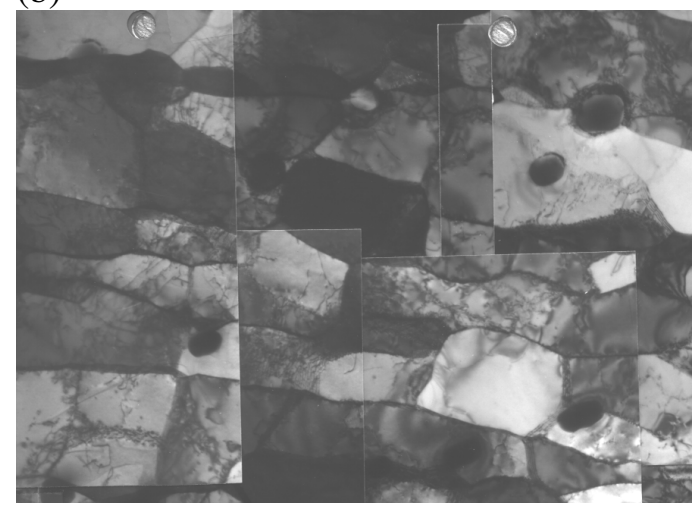

Figure 3: Dislocation substructures in Al$\% M g$ during thermomechanical processing: (a) elongated microbands after deformation at $300^{\circ} \mathrm{C}$ and $0.1 / \mathrm{s}$ to a strain of $0.3 \mathrm{in}$ axisymmetric tension, (b) cells within elongated microbands after deformation at $385^{\circ} \mathrm{C}$ and $2.5 / \mathrm{s}$ to a strain of 0.7 in plane strain compression and (c) equiaxed subgrains after deformation in plane strain compression at $385^{\circ} \mathrm{C}$ and $2.5 / \mathrm{s}$ to a strain of unity in plane strain compression testing. $(b \quad \& \quad c)$ samples are taken from the maximum deformation zone, while in (a) a homogenous tension deformation means the sample can be taken from anywhere. 
(a)

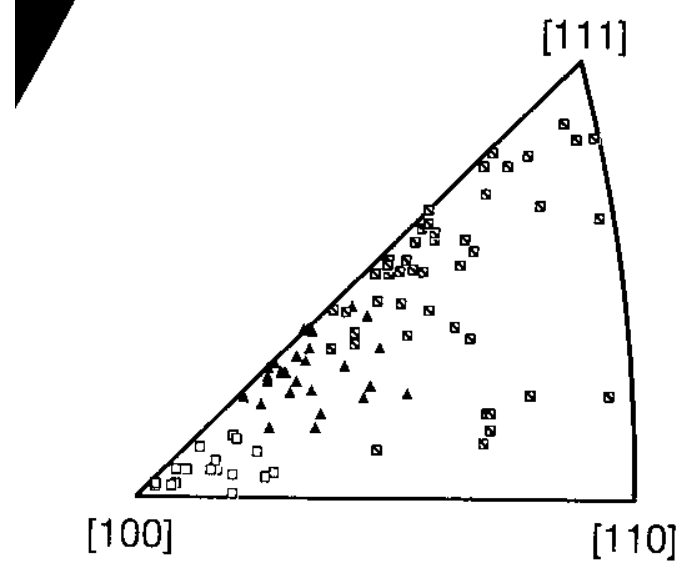

(c)

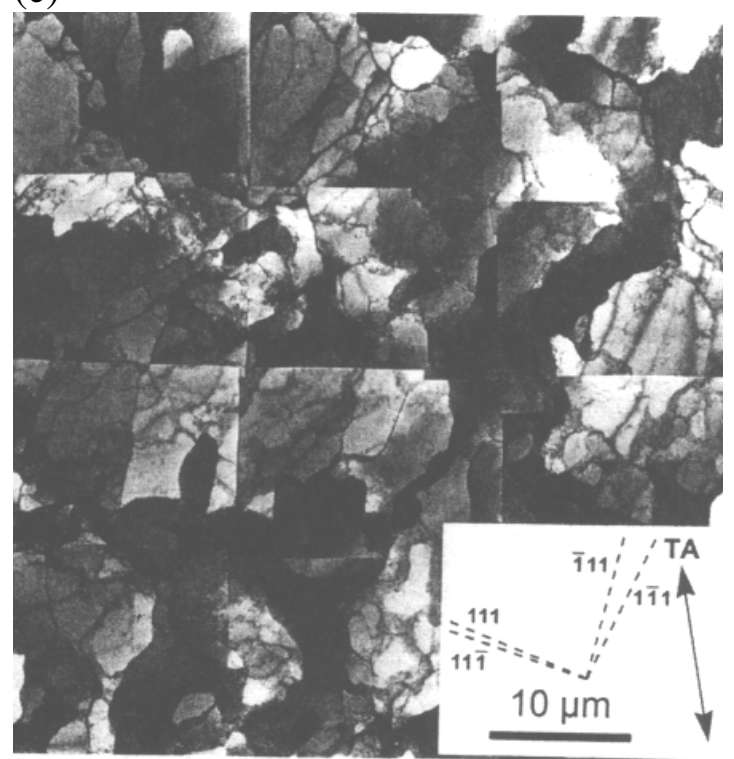

(b)

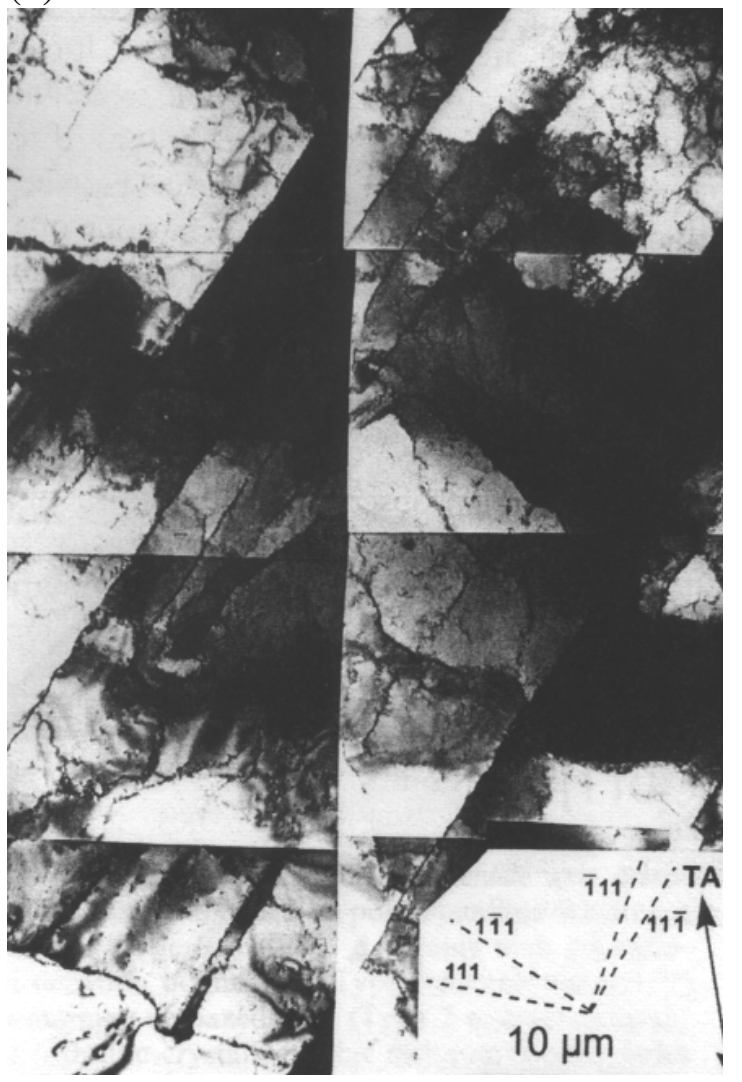

(d)

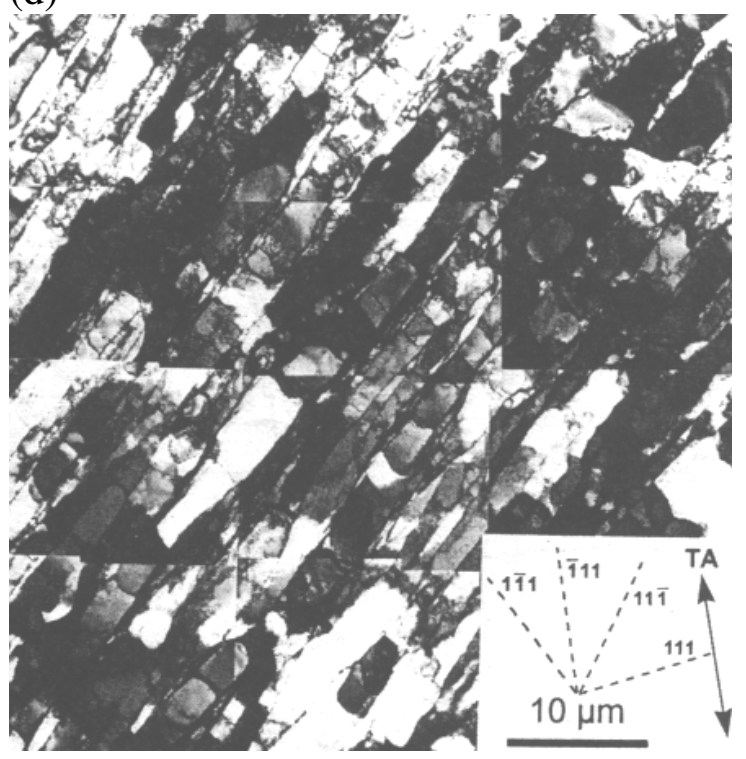

Figure 4: Dislocation substructures of pure aluminium after room temperature tension at a strain rate of $8.3 \times 10^{-4} / \mathrm{s}:$ (a) Inverse pole figure showing the tensile axis orientation of 89 grains embedded in polycrystalline specimens strained in the range 0.05-0.34. (b) Type 1 represents the grains with crystallographic dislocation boundaries, (c) type 2 the grains containing equiaxed dislocation cell/subgrains and (d) type 3 the grains with noncrystallographic dislocation boundaries. 

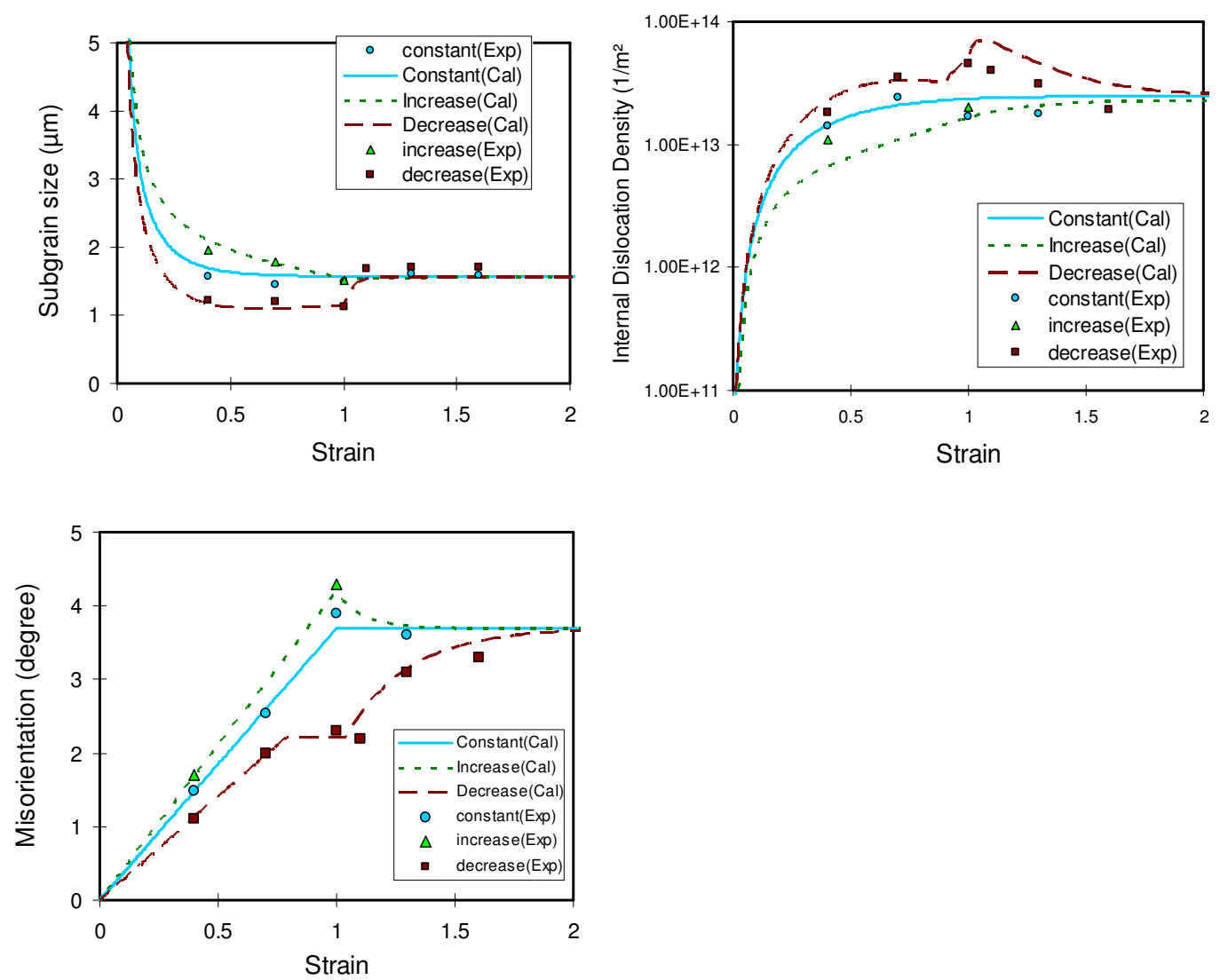

Figure 5: Internal states evolution using the neuro-fuzzy model prediction for Al-1\%Mg material. 


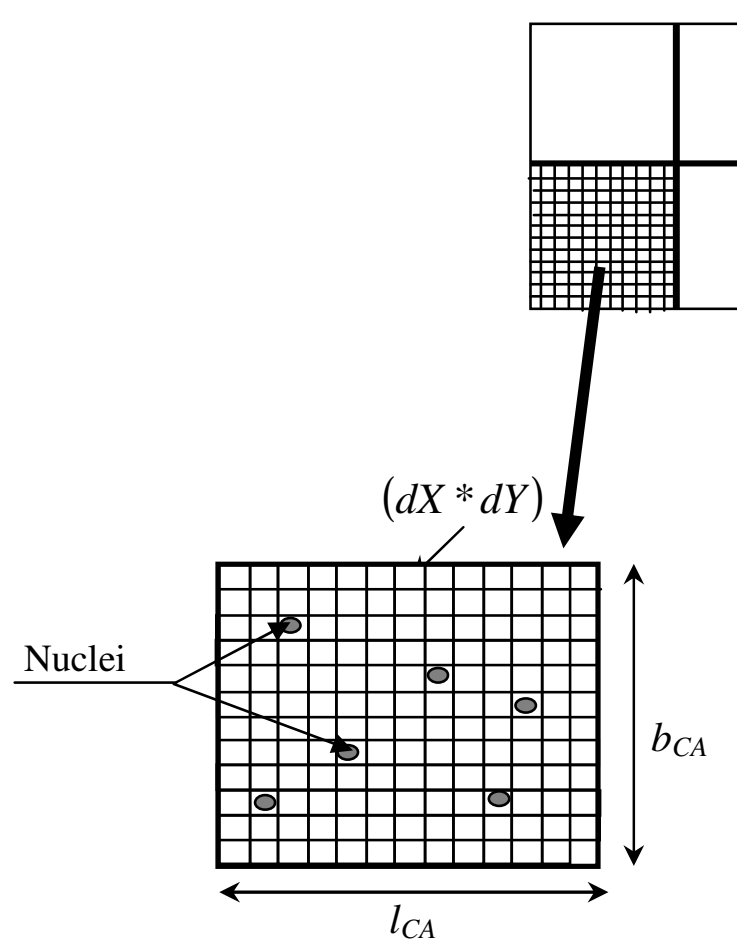

(b)

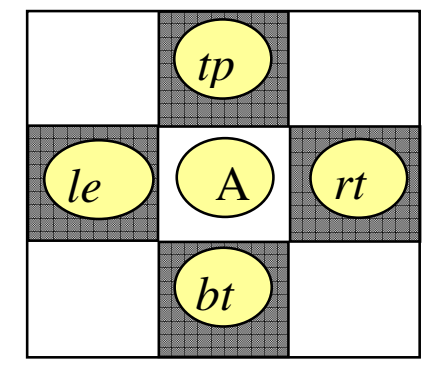

(d)

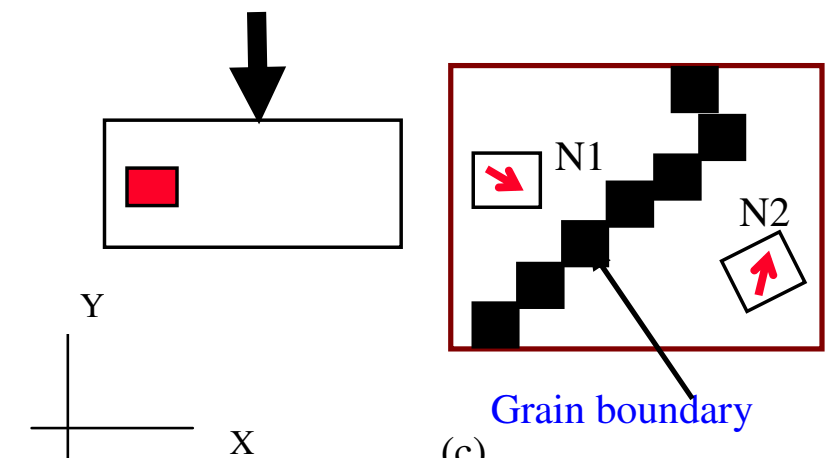

(c)

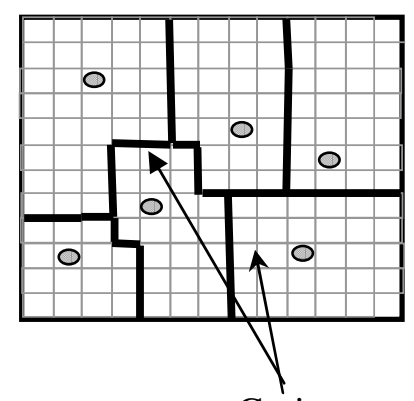

Grains

(e)

Figure 6: The three domains of the present CAFE model. (a) The structure domain divided into a number of finite elements; one element is shown with its expected array of cells. (b) A population of nuclei in a CA array from which the grain will grow. (c) Definition of orientations with respect to the loading direction. (d) Moore's definition of a CA neighbourhood. (e) A grain structure that emerges from the nuclei of (b). 


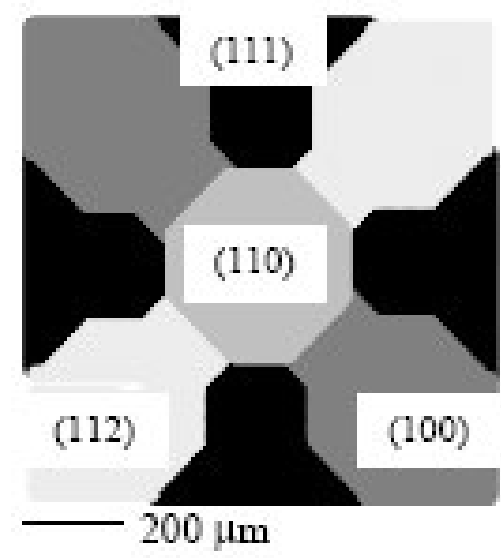

(a) simulated microstructure.

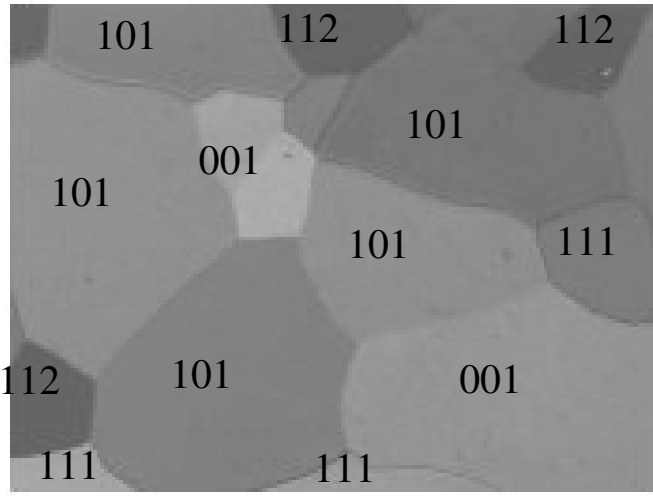

(b) experimental microstructure.

Figure 7: Simple microstructure with 4 orientations. 


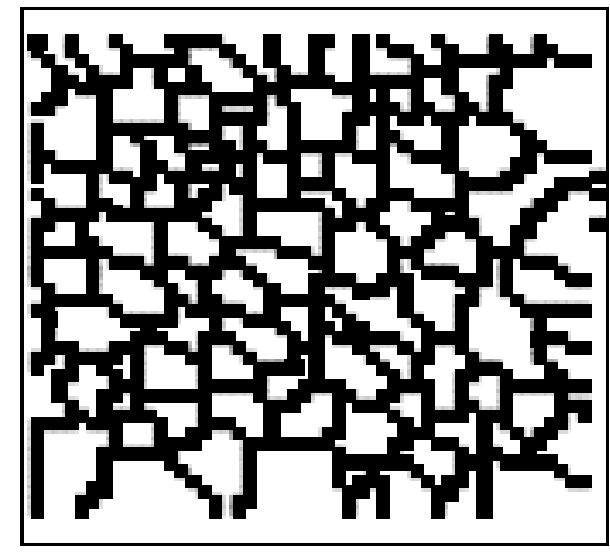

$200 \mu \mathrm{m}$

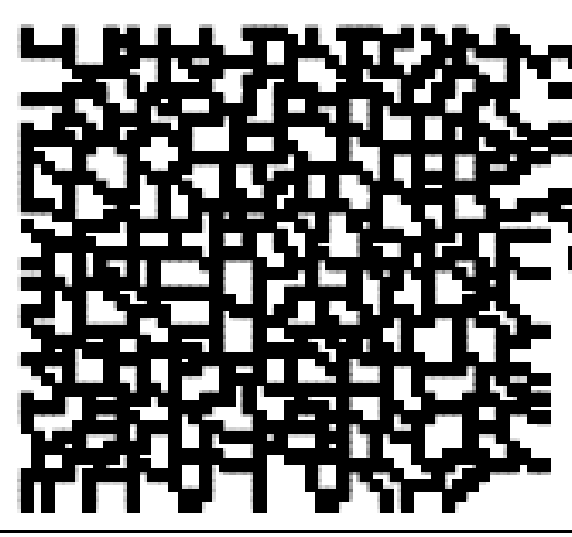

$200 \mu \mathrm{m}$

Figure 8: Representative microstructures with (a) 100 grains forming a coarse microstructure. (b) 300 grains forming a fine microstructure. 

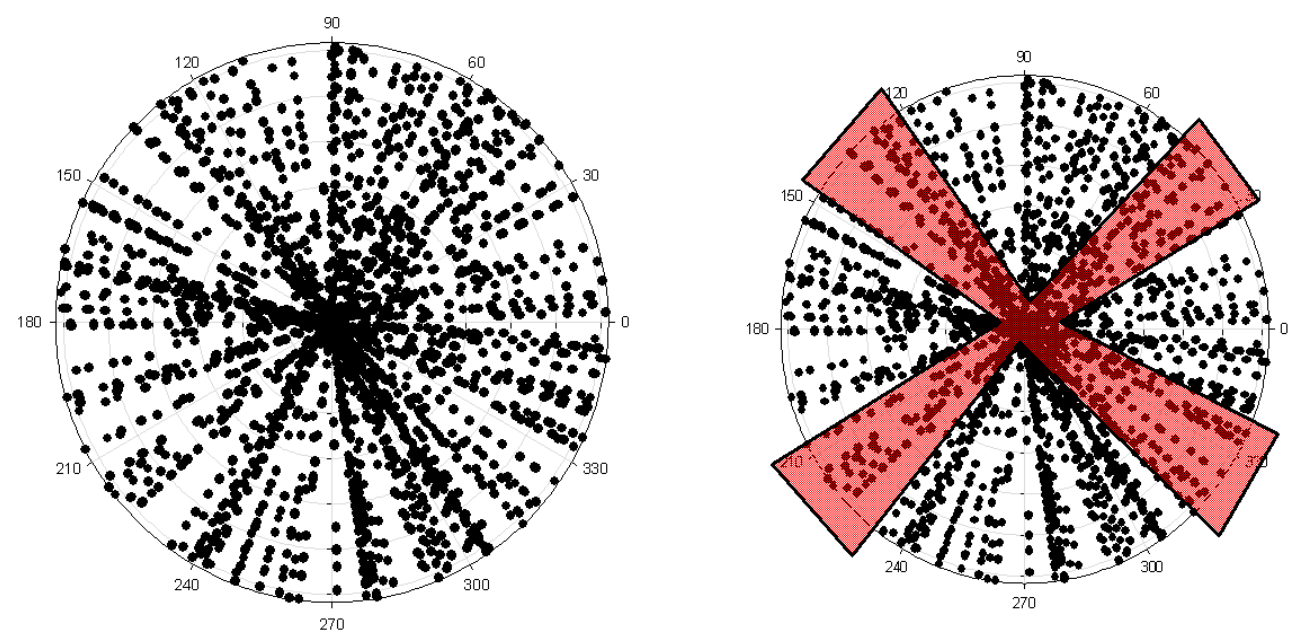

Figure 9: (a) Orientation distributions for each nucleus used to generate the microstructure of Figure 7. (b) Highlighted region shows biasing of specific orientations with respect to a particular loading direction. 


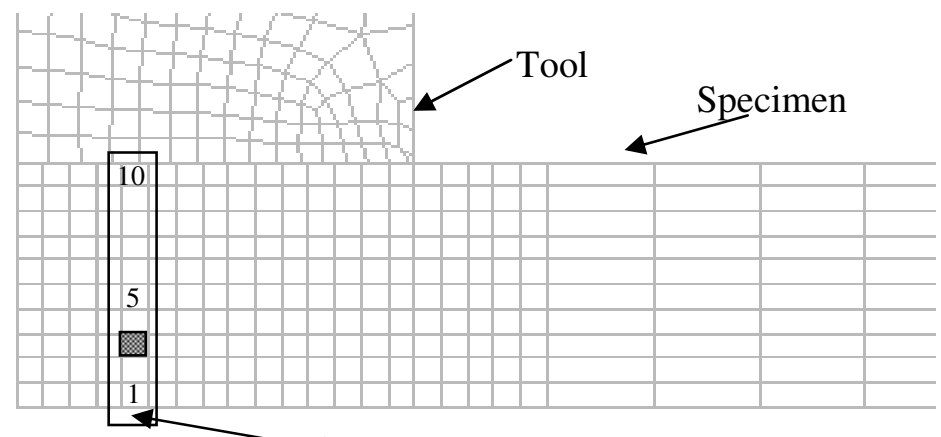

Element set $\mathrm{A}$

Figure 10: Finite element model assembly for the PSC tests. 


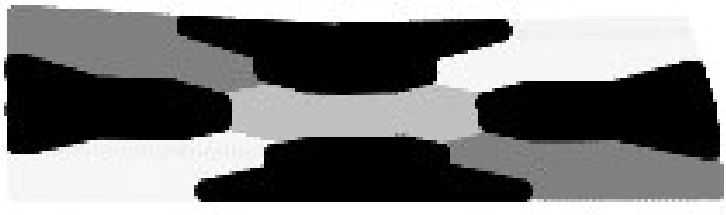

$200 \mu \mathrm{m}$

(a) simulated microstructure.

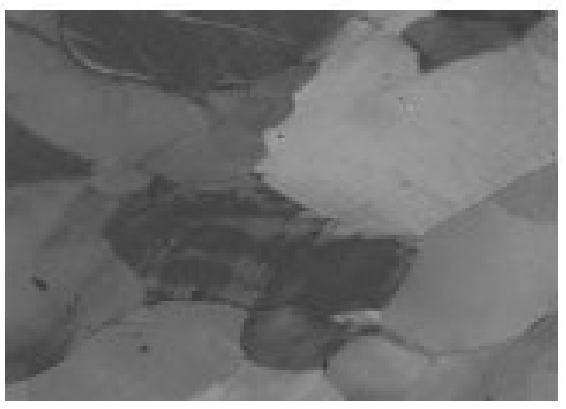

(b) experimental microstructure.

Figure 11: The simple microstructure of figure 3 after deformation. 


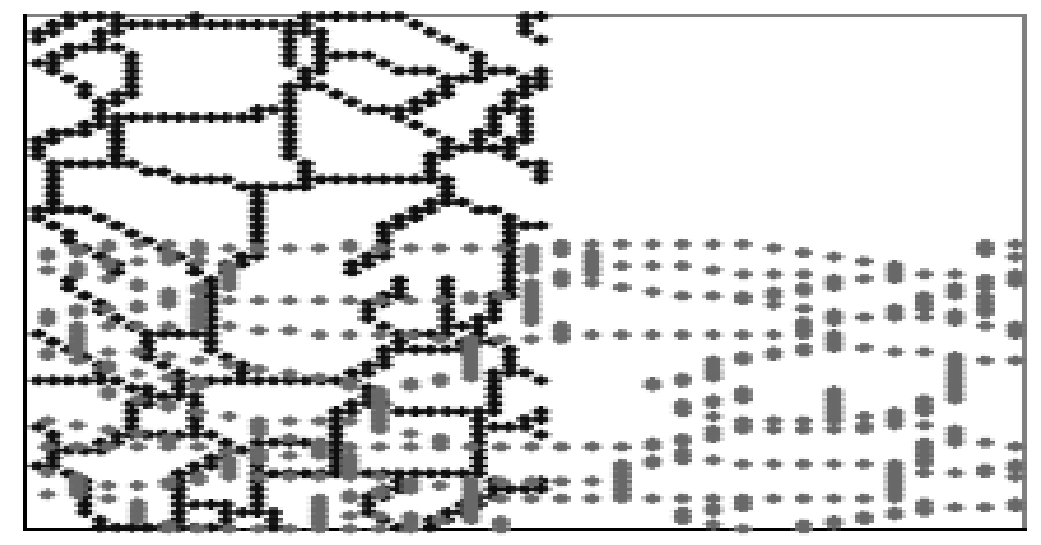

Figure 12: Change in microstructure shape for element no. 3, before and after deformation. 


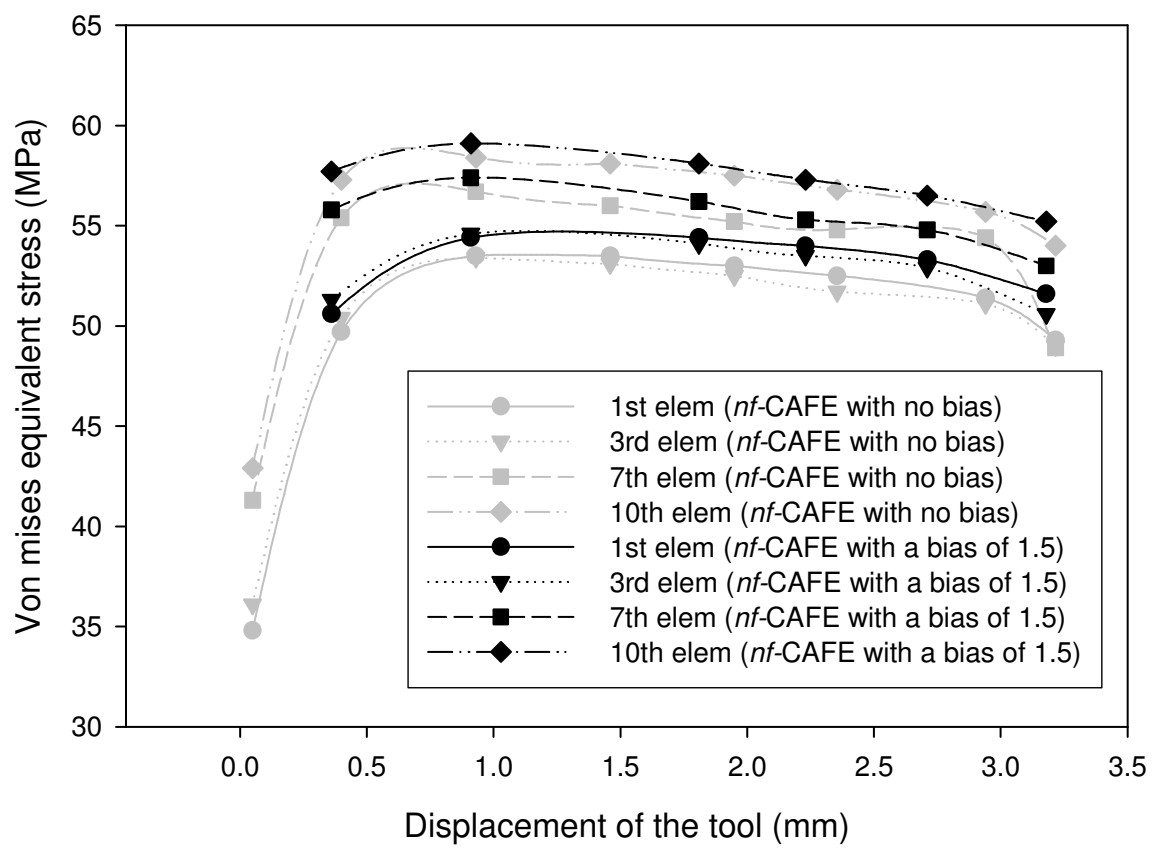

(a) comparison of von Mises stress for 1.5 bias.

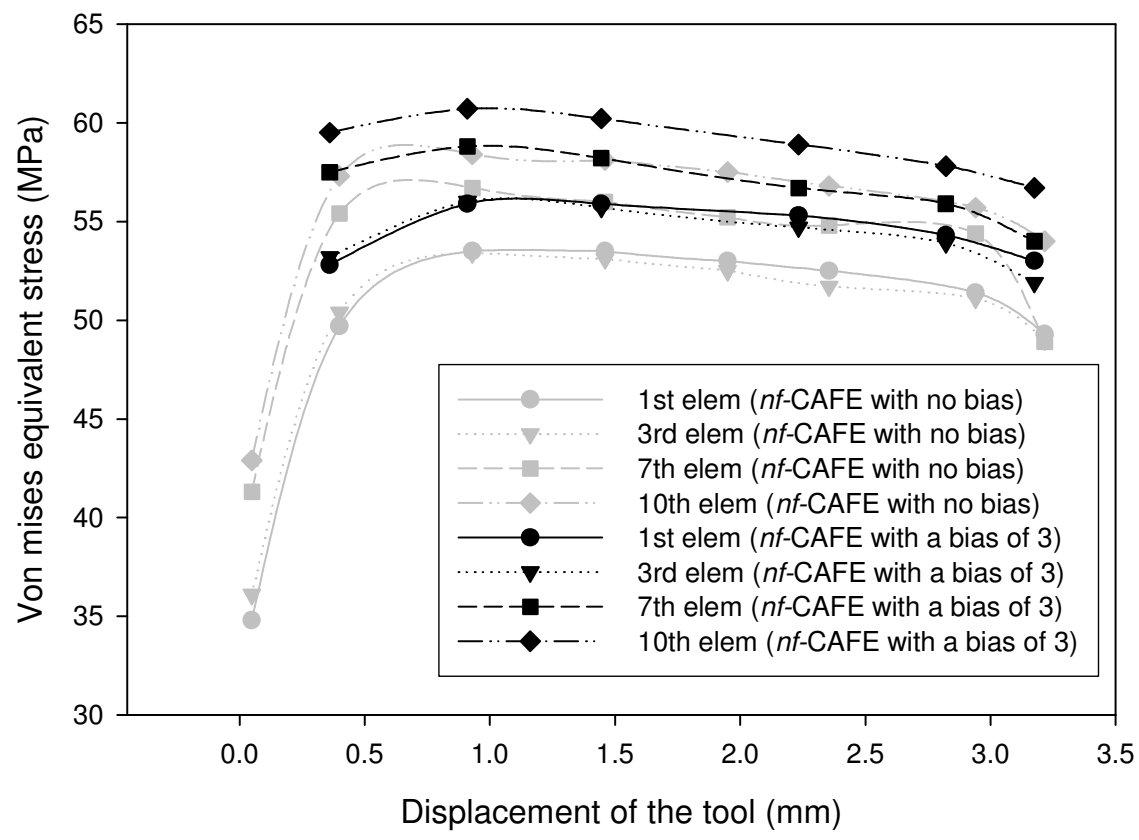

(b) comparison of von Mises stress for 3.0 bias.

Figure 13: von Mises stress distribution to show the effect of strain biasing at grain boundaries using the $n f$-CAFE methodology 


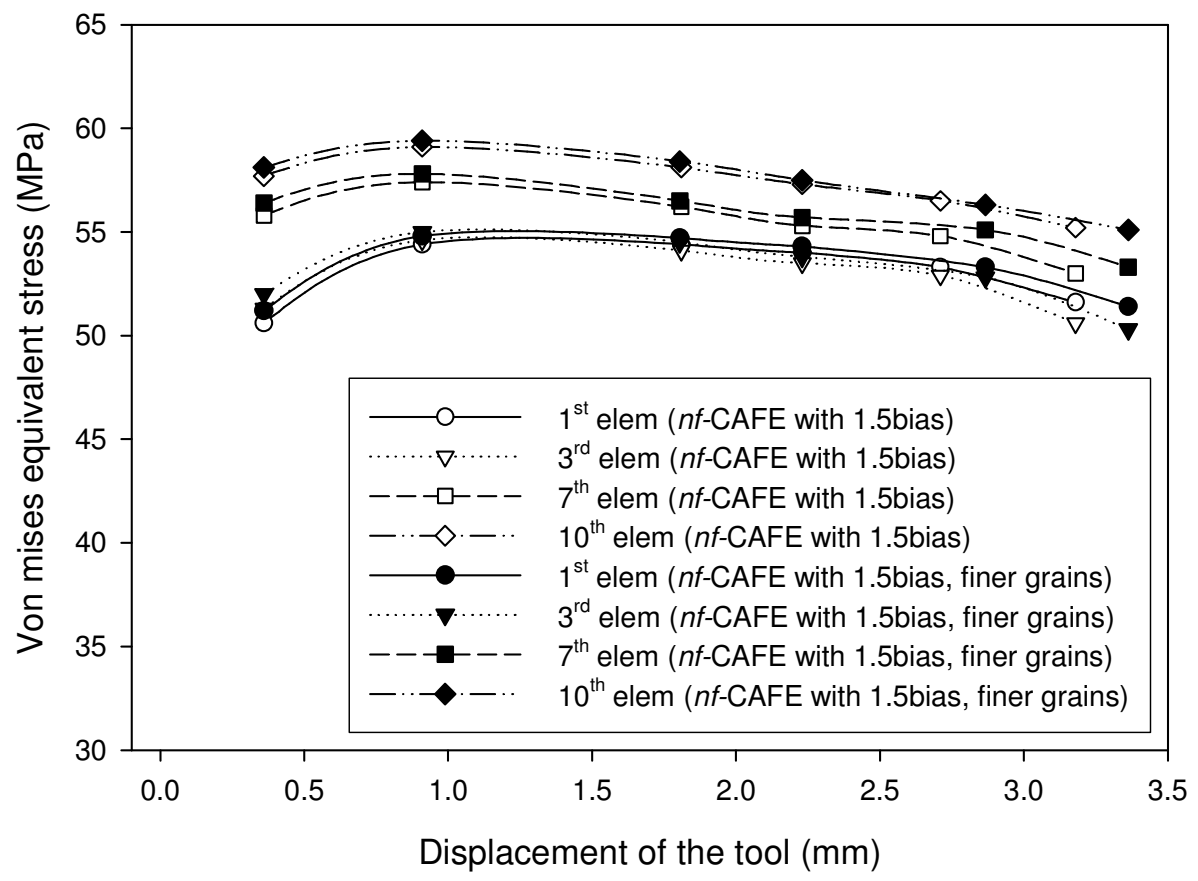

Figure 14: Comparison of nf-CAFE model prediction with a change in the grain size. 


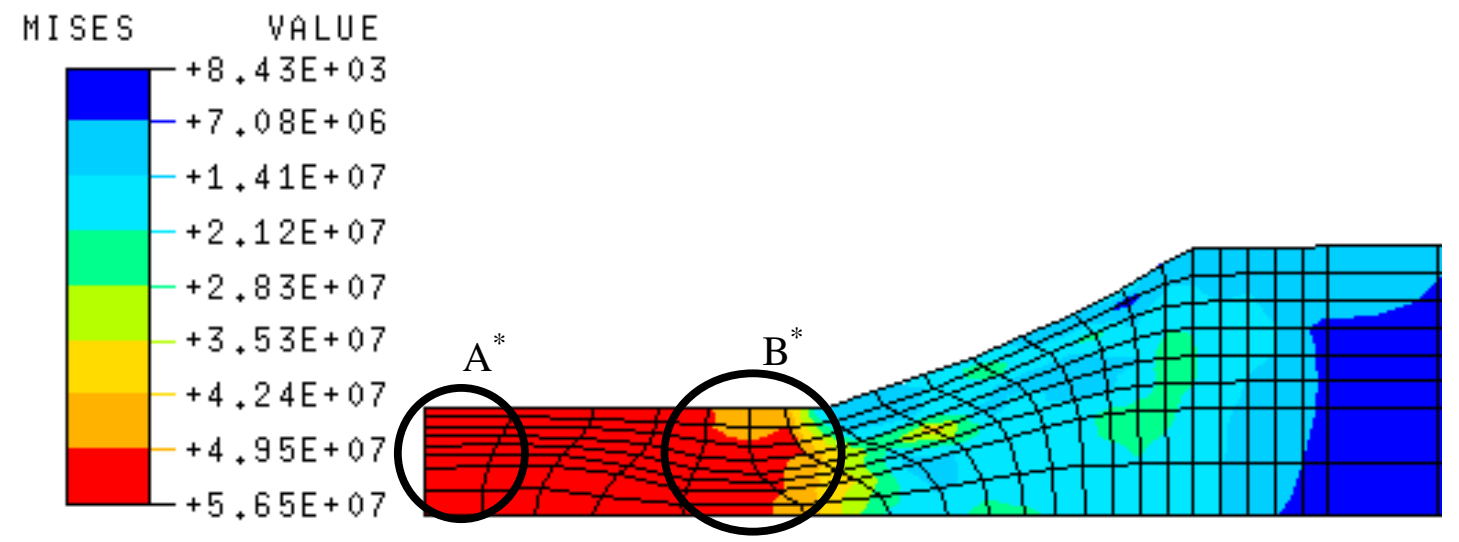

(a)
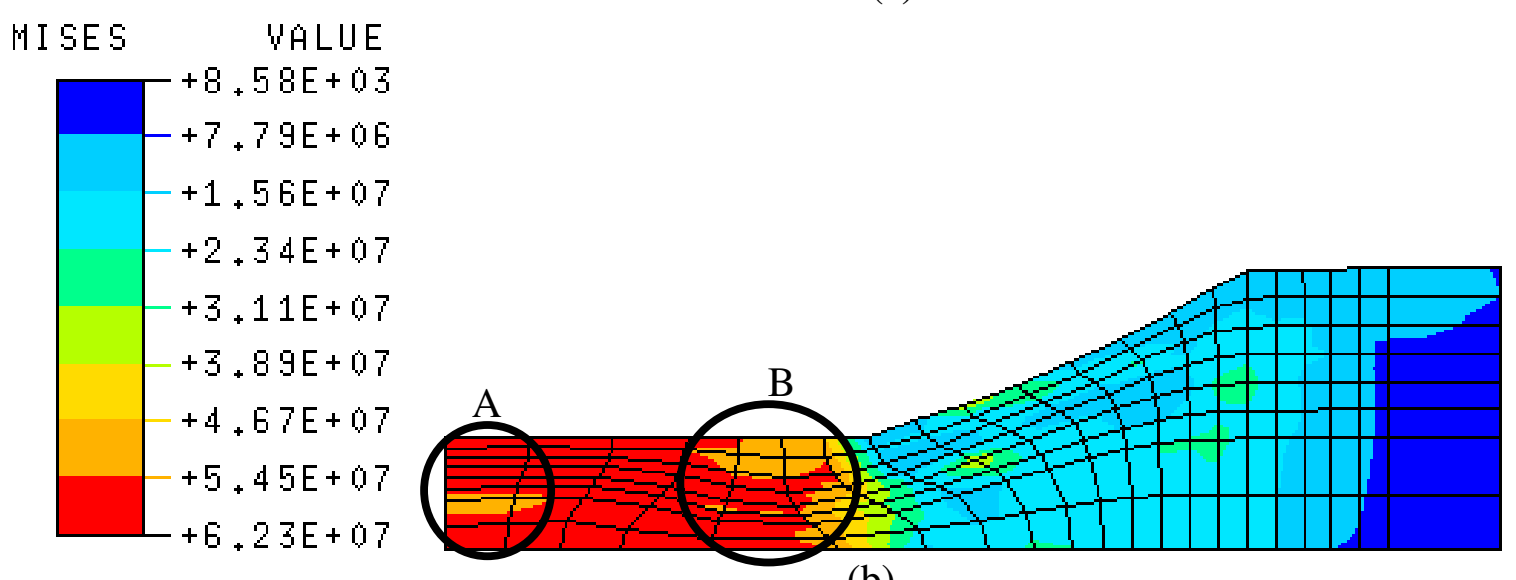

(b)

Figure 15: Comparison of nf-CAFE model prediction with introduction of orientation biasing. (a) No Biasing. (b) With a biasing of $20 \%$. 